\title{
Integrating species and interactions into similarity metrics: a graph theory-based approach to understanding community similarity
}

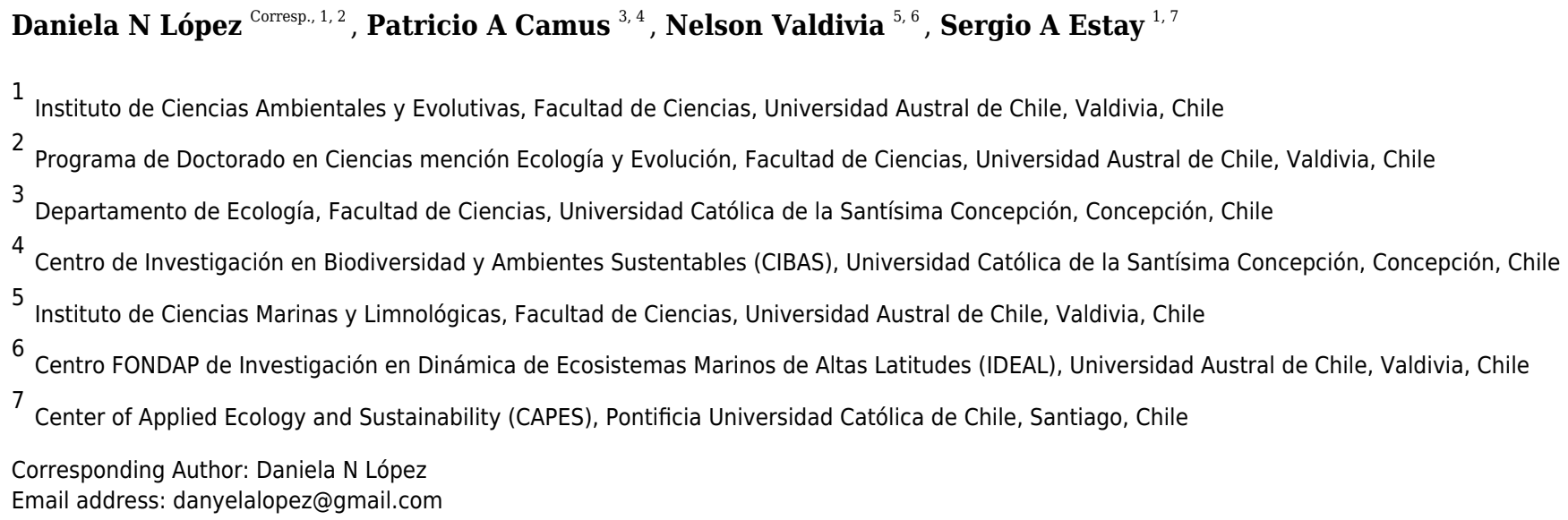

Community similarity is often assessed through similarities in species occurrences and abundances (i. e. compositional similarity) or through the distribution of species interactions (i. e. interaction similarity). Unfortunately, the joint empirical evaluation of both is still a challenge. Here, we analyze community similarity in ecological systems in order to evaluate the extent to which indices based exclusively on species composition differ from those that incorporate species interactions. Borrowing tools from graph theory, we compared the classic Jaccard index with the graph edit distance (GED), a metric that allowed us to combine species composition and interactions. We found that similarity measures computed using only taxonomic composition could differ strongly from those that include composition and interactions. We conclude that new indices that incorporate community features beyond composition will be more robust for assessing similitude between natural systems than those purely based on species occurrences. Our results have therefore important conceptual and practical consequences for the analysis of ecological communities. 
1 Integrating species and interactions into similarity metrics: a graph theory-based approach to

2 understanding community similarity

3

4 López, Daniela $\mathrm{N}^{1,2}$, Camus, Patricio $\mathrm{A}^{3,4}$, Valdivia, Nelson ${ }^{5,6}$ \& Estay, Sergio A $\mathrm{A}^{1,7}$

5

$6{ }^{1}$ Instituto de Ciencias Ambientales y Evolutivas, Facultad de Ciencias, Universidad Austral de 7 Chile, Valdivia, Chile.

$8{ }^{2}$ Programa de Doctorado en Ciencias mención Ecología y Evolución, Facultad de Ciencias, 9 Universidad Austral de Chile, Valdivia, Chile.

$10{ }^{3}$ Departamento de Ecología, Facultad de Ciencias, Universidad Católica de la Santísima

11 Concepción, Concepción, Chile.

$12{ }^{4}$ Centro de Investigación en Biodiversidad y Ambientes Sustentables (CIBAS), Universidad

13 Católica de la Santísima Concepción, Concepción, Chile.

$14{ }^{5}$ Instituto de Ciencias Marinas y Limnológicas, Facultad de Ciencias, Universidad Austral de 15 Chile, Valdivia, Chile.

$16{ }^{6}$ Centro FONDAP de Investigación en Dinámica de Ecosistemas Marinos de Altas Latitudes 17 (IDEAL), Universidad Austral de Chile, Valdivia, Chile.

$18{ }^{7}$ Center of Applied Ecology and Sustainability (CAPES), Pontificia Universidad Católica de 19 Chile. Santiago, Chile.

21 Corresponding author: Daniela N. López, Instituto de Ciencias Ambientales y Evolutivas, 22 Facultad de Ciencias, Universidad Austral de Chile. Edificio Emilio Pugín, tercer piso oficina 23 313, Valdivia, Chile.

24 Telephone number: $056+63+2221344$ 
25 Correspondence: E-mail: danyelalopez@gmail.com.

27 Abstract

28 Community similarity is often assessed through similarities in species occurrences and

29 abundances (i. e. compositional similarity) or through the distribution of species interactions (i.

30 e. interaction similarity). Unfortunately, the joint empirical evaluation of both is still a challenge.

31 Here, we analyze community similarity in ecological systems in order to evaluate the extent to

32 which indices based exclusively on species composition differ from those that incorporate

33 species interactions. Borrowing tools from graph theory, we compared the classic Jaccard index

34 with the graph edit distance (GED), a metric that allowed us to combine species composition and

35 interactions. We found that similarity measures computed using only taxonomic composition

36 could differ strongly from those that include composition and interactions. We conclude that new

37 indices that incorporate community features beyond composition will be more robust for

38 assessing similitude between natural systems than those purely based on species occurrences.

39 Our results have therefore important conceptual and practical consequences for the analysis of

40 ecological communities. 
41

42

43

44

\section{Introduction}

Characterizing the degree of similarity between ecological communities has been one of the central topics in ecology (Devictor et al., 2010; Morlon et al., 2014; Petchey and Gaston, 2006). In most studies, ecological similarity has mainly been evaluated by comparing species compositions, quantifying the spatial turnover of species through cluster membership of communities and by examining the position and distance between communities in reduced species-ordination spaces (Jaillard et al., 2018; Olden et al., 2004). In this vein, $\beta$-diversity portrays the variation in species composition among spatially or temporally separated communities (Anderson et al., 2006; Whittaker, 1972, 1960), and thus reflects two different phenomena, spatial species turnover and nestedness of assemblages (Baselga, 2012, 2010). $\beta$ diversity can be easily measured from species presence-absence data (Koleff et al., 2003; Wilson and Shmida, 1984) or abundances (Barwell et al., 2015), and both approaches include some widely studied indices such as Euclidean distances, Bray-Curtis, and Jaccard (Anderson et al., 2006). Despite the widespread use of $\beta$-diversity and other indices to characterize communities, the quantification of similarity among ecological communities, beyond species compositions remains a challenge.

In an analogous way, when ecological communities are represented by interaction networks, the similarity between spatially or temporally separated communities has been evaluated on the basis of the average number of shared nodes (species) and the degree of node overlapping (i.e. node overlapping index; Strona and Veech, 2015; Strona et al. 2018; Zhang et al., 2016). These measures assume that a high compositional similarity implies a high similarity in species interactions (i.e. a high "interaction similarity"). On the other hand, it has been described that species composition can be a driver of interaction turnover in mutualistic networks 
64 (Bezemer et al., 2010; Trøjelsgaard et al., 2015), but there is also evidence based on traditional

65 methods ( $\beta$-diversity) that shows a complete lack of correlation between composition similarity

66 and interaction similarity (Poisot et al., 2012). Therefore, the relationship between composition

67 and interaction similarity remains unclear.

Graph theory provides us with conceptual and practical tools that allow us to integrate the composition and interaction measurements of similarities of systems (Ibragimov et al., 2013;

Riesen, 2016). These similarity measures include global metrics based on isomorphic relations or on graph transformations (Bunke and Allermann, 1983; Dehmer, 2010; Emmert-Streib et al., 2016; Solé-Ribalta et al., 2012). Similarity measures based on isomorphic relationships, quantify the exact match of nodes and links between two graphs, i.e. the "exact graph matching”. Graph transformation, on the other hand, uses the concept of error-tolerant graph matching to calculate a measure of similarity based on the minimum cost (i.e. a unit of dissimilarity) of transforming one graph into another (Dehmer, 2010; Riesen, 2016). A widely used graph transformation method is the graph edit distance (GED), in which each transformation has a cost, so that a greater number of changes mirrors higher dissimilarity between the analyzed networks (Bunke and Allermann, 1983; Emmert-Streib et al., 2016). In biological sciences, GED has been used to compare protein-protein interaction (PPI) networks in human, yeast, and fruit fly, allowing

81 researchers to infer the biological function of proteins and genes (Ibragimov et al., 2013;

82 Neyshabur et al., 2013). By means of applying these tools to ecological data we can obtain

83 indices that include both species composition and species interactions, providing a more 84 complete view of community similarity. In this study we apply conceptual tools borrowed from graph analysis to quantify

86 community similarity, taking into account both species composition and species interactions. In 
87 addition, we compare the inferences obtained from this approach with those obtained using the

88 classical compositional approach in order to evaluate the degree to which interaction similarity

89 can be inferred from compositional similarity. These analyses were applied to an extensive

90 consumer-resource interaction network.

92 2. Materials and methods

\subsection{Biological data}

94 We analyzed a dataset (https://www.nceas.ucsb.edu/interactionweb/html/thomps

95 towns.html) of consumer-resource interactions obtained from the National Center for

96 Ecological Analysis and Synthesis (NCEAS). The dataset includes 16 communities (Akatore A,

97 Berwick, Blackrock, Broad, Canton, Catlins, DempSp, German, Kyeburn, Narrowdale,

98 NorthCol, Powder, Stony, SuttonSp, and Venlaw), covering ca. $200 \mathrm{~km}$ of the Taieri River

99 tributaries in Otago, New Zealand. These sites include pine forest, broadleaf forest, pasture

100 grassland, and tussock grassland with recorded taxonomic identities of aquatic insects, algae, and

101 fish species and their trophic interactions (Jaarsma et al., 1998; Thompson and Townsend, 2005,

102 2003, 2000, 1999). The size of these networks varies from 48 to 113 taxonomic identities and

103 from 110 to 832 consumer-resource interactions. We assign an identification code to taxonomic

104 identities to facilitate comparison among different networks. We made some modifications to the

105 network dataset prior the analyses (see the Data S1), checking scientific names and correcting

106 typographical errors. Finally, similarity measures were calculated for paired combinations of

107 sites in Taieri river (120 paired comparisons for each scenario).

108

109 2.2. Graph edit distance (GED): concept and application 
We used the graph edit distance (GED) as a metric that includes both compositional and

111 interaction similarities. GED is a widely used graph transformation method in which each

112 transformation (edition) necessary to pass from one network to another has a cost, so that a

113 greater number of changes implies a higher cost, and this mirrors higher dissimilarity between

114 the compared communities (Bunke, 1983; Emmert-Streib et al., 2016). This feature of GED

115 represents an advantage over the composition similarity analysis, because it allows to include

116 interaction similarity in the metrics and to assign different degrees of importance to species or

117 interactions in the network through differential costs for each type of edition (Bunke and

118 Allermann, 1983; Emmert-Streib et al., 2016). Despite that our study only deals with trophic

119 interactions, GED could be applied to networks containing different kinds of links, including

120 other ecological interactions like facilitation, competition, and parasitism.

121 Let us consider two networks represented by the graphs $g_{1}=\left(V_{1}, E_{1}\right)$ and $g_{2}=\left(V_{2}, E_{2}\right)$,

122 where $\mathrm{V}$ is a set of nodes, $\mathrm{E}$ a set of links $(\mathrm{u}, \mathrm{v})$, where $\mathrm{u} \in \mathrm{V}$ is the source node and $\mathrm{v} \in \mathrm{V}$ the

123 target node of a directed link (Dehmer, 2010; Riesen, 2016). The idea behind GED includes

124 transforming one graph into another using edit operations $\left(e_{i}\right)$ such as deletions, substitutions,

125 and insertions of nodes and links (Bunke and Allermann, 1983; Dehmer, 2010; Emmert-Streib et

126 al., 2016; Riesen, 2016). A given transformation is represented by an edit path ( $\lambda$ ), which is a set

127 of edit operations that transform $g_{1}$ to $g_{2}$. The set of all possible $\lambda$ is called $Y\left(g_{1}, g_{2}\right)$ (Riesen,

128 2016). Assuming each edit operation $e_{i}$ has an associated $\operatorname{cost} c\left(e_{i}\right)$, we can assign a relative cost

129 to the $\mathrm{k}^{\text {th }}$ edit path $\lambda_{\mathrm{k}}$ :

$130 \quad \mathrm{C}\left(\lambda_{\mathrm{k}}\right)=\sum \mathrm{c}\left(e_{i}\right), \forall e_{i} \in \lambda_{\mathrm{k}}$

131 With this information we can define GED as the edit path with the minimum cost

132 (Dehmer, 2010; Ibragimov et al., 2013), 


$$
G E D=\min \left[C\left(\lambda_{k}\right)\right], \forall \lambda_{k} \in Y(g 1, g 2)
$$

134 The application of GED to the study of trophic networks allows us to evaluate differences

135 in species compositions and those due to the absence of interactions despite species co-

136 occurrences.

\subsection{Cost operations}

Given the nature of food webs, we considered that the lowest cost was that of deleting or

140 adding an interaction, followed by deleting or adding a species, and finally the most costly

141 edition was that of flipping an interaction, this is a change the hierarchy of the consumer-

142 resource interactions (Table 1 , scenarios 30, 35, 36). We considered 49 scenarios of editing costs

143 (8820 paired comparisons of our networks), which differed from each other in the relative

144 magnitude of the costs of each edition operation $c\left(e_{i}\right)$, as shown on the Table 1 . We include two

145 cost scenarios of flipping an interaction (0.25 and 0.75$)$ because represent two contrasting

146 scenarios (low and high cost), and 5 levels of costs for deleting/adding an interaction or node.

147 Also, eight scenarios were included specifically to assess how much GED changed by

148 minimizing the importance of trophic interactions (reducing the costs to deleting or adding

149 interactions, Table 1).

150 GEDs were calculated using the software Cytoscape (Shannon et al., 2003) with the GEDEVO

151 plugin (Ibragimov et al., 2013), which implements an evolutionary algorithm for GED

152 calculation. Due to GEDEVO implements a evolutionary algorithm for estimate GED, there is no

153 a straightforward rule for stopping the iterative process. In this case we used 1,000 iterations

154 without improvements as termination criterion for the minimization of GED. In the case of

155 GEDEVO, GED scores ranged from 0 to 1 , where 0 represented maximum similarity and 1 
156 represented perfect dissimilarity. According to Malek (2015), the method implemented in

157 GEDEVO consists in that the GED of a given edit path is the result of the sum of the GED

158 estimated for each node. The GED form each node is transformed into a score based on the

159 highest possible GED for a single node in the whole network.

160

161 2.4. Compositional similarity and comparison with GED scores

162 In order to compare the GED results with those from a traditional compositional

163 approach, we first computed the Jaccard index from the presence/absence species data of each

164 network. Jaccard dissimilarity index was computed as $(a+b) /(a+b+c)$, where $a$ is the number of

165 species only present in the first network, $b$ is the number of species only present in the second

166 network, and $c$ is the number of species shared between both networks.

167 In our results, the GED and Jaccard scores were expressed in terms of similarity (i.e. 1 -

168 GED and 1 - Jaccard, respectively). We used the Adjusted Mutual Information metric (AMI) to 169 assess the amount of information shared between GED and Jaccard.

170 Mutual Information (MI) is an measure from information theory and based on entropy

171 estimations. MI quantifies the mutual dependence between the variables or, in other words, how

172 much information about one variable is possible to obtain by knowing the second variable. MI

173 can theoretically range from 0 to $\infty$. So, to standardize the values and make them comparable we

174 used the method from Vinh et al. (2010). In this case AMI is:

175

$$
A M I_{\max }=I(U, V)-E\{I(U, V)\} / \max \{H(U), H(V)\}-E\{I(U, V)\}
$$

176

177 where, $U$ and $V$ are two dataset, $I(U, V)$ represents to mutual information, $E\{I(U, V)\}$ the

178 expected mutual information, and finally $H(U, V)$ joint entropy, thus: 
$179 I(\mathrm{U}, \mathrm{V})=\sum_{u \in U} \sum_{v \in V} p(\mathrm{u}, \mathrm{v}) \log p(\mathrm{u}, \mathrm{v}) / p(u) p(v)$,

$180 H(\mathrm{U}, \mathrm{V})=-\sum_{u \in U} \sum_{v \in V} p(\mathrm{u}, \mathrm{v}) \log p(\mathrm{u}, \mathrm{v})$, where the entropy of a random variable is defined by:

$181 H(U)=-\sum p(u) \log p(u)$ or

$182 H(V)=-\sum p(v) \log p(v)$,

183 with probability functions $p(u)$ and $p(v)$.

184 The advantage of AMI is that it allows us to quantify linear and non-linear relationship

185 between variables. AMI scores ranged from 0 to 1 , where 0 represented perfect independence (no

186 information is shared between indices) and 1 means both variables contain exactly the same

187 information (Vinh et al. 2010). Jaccard index and AMI calculations were performed in the R

188 environment (R Core Team, 2016, Data S2). Jaccard index values were calculated using vegan

189 package for R (Oksanen et al. 2013) and AMI values were calculated using the function

190 discretize from the R package infotheo (Meyer 2014).

191 We used the same approach to compare dissimilarity of interactions $\left(\mathrm{B}_{\mathrm{WN}}\right)$ and the

192 dissimilarity of interactions due to species turnover $\left(\mathrm{B}_{\mathrm{ST}}\right)$ as there are defined in Poisot et al.

193 (2012) with the estimated GED values in the 49 scenarios (Table 2). $\mathrm{B}_{\mathrm{WN}}$ and $\mathrm{B}_{\mathrm{ST}}$ values were

194 calculated using betalink package for R (Poisot et al. 2012). Finally, we used the AMI to

195 compared the amount of information shared between GED and $\mathrm{B}_{\mathrm{WN}}$, and between GED and $\mathrm{B}_{\mathrm{ST}}$.

196 The objective of this comparison is to evaluate the relationship of GED with another recent

197 methodological approach to the problem, and also to evaluate the relationship of GED under

198 different cost scenarios with the result obtained by partitioning network similarity into species

199 and interaction $\beta$-diversity as is proposed by Canard (2011) and Poisot et al. (2012).

200 3. Results 
The interaction similarity scores based on the GED ranged from 0.099 to 0.99 for the

202 Taieri river networks. On the other hand, compositional similarity (Jaccard) ranged from 0.08 to

203 0.67. The highest values of GED were found when the interactions had zero cost (Figs. 1A). We

204 found lower values of GED when the adding/deleting nodes had zero cost and adding/deleting

205 interactions had a low cost (Figs.1C, 1D, Table 1). A general pattern in the relationship between

206 Jaccard and GED can be observed in Fig. S1. Through almost all scenarios, at low levels of the

207 Jaccard index $(<0.2)$, estimated GED values tend to be highly variable, but at moderate-high

208 values $(>0.2)$ the relationship turns asymptotic (Figs. 1C, 1D, S1). Due to this relationship, AMI

209 values were low, regardless of the scenario. Estimated values were far from 1, with a maximum

210 of 0.134 for scenario 2 and minimum of 0.014 for scenario 35 (Fig. 2, Table 1). The

211 interpretation of these markedly low AMI values irrespective of the scenario means both metrics

212 seem to be unrelated and do not share significant amounts of information (see Fig. 2). In

213 practical terms, network similarity (nodes and links) cannot be inferred or predicted from

214 similitude at the species level.

215 We also found a weak relationship between $\mathrm{B}_{\mathrm{ST}} / \mathrm{B}_{\mathrm{WN}}$ and GED across all scenarios (Fig.

216 3, Table 2). Despite of this, AMI values were consistently higher for the relationship between

$217 \mathrm{~B}_{\mathrm{WN}}$ and GED (AMI from 0.008 to 0.156 ) than between $\mathrm{B}_{\mathrm{ST}}$ and GED (AMI from $\sim 0$ to 0.029 ).

218 The maximum AMI value was observed when the cost of adding/deleting nodes was lower than

219 adding/deleting links (Table 2).

220

221

222 4. Discussion 
224 Jaccard and GED indices; the AMI values were far from those expected for both metrics equally

225 describing the communities. Similar results have been described by other studies in which

226 differences between similarity metrics (composition similarity - interaction similarity) has been

227 explained by phenomena such as changes in phenology (Edwards and Richardson, 2004), trophic

228 interactions mediated by the presence of a third species, and changes in abundance, behavior, or

229 physiology (Burkle et al., 2013; Poisot et al., 2012). Undoubtedly, these phenomena confer

230 variation to communities in different ways, and thus affect the occurrence of both species and

231 trophic interactions. In the same vein, changes in climatic conditions can decouple the synchrony

232 of ecological processes, such as predator-prey interactions (Harrington et al., 1999; Thackeray et

233 al., 2010). This implies that short-term changes in species' seasonal phenology could result in the

234 loss of some predator-prey interactions, thus impacting interaction similarities. Likewise,

235 mechanisms such as changes in species abundances and predator behavior can jointly affect the

236 trophic similarity of communities (Abrams, 1982; Arditi and Ginzburg, 2012). Examples of these

237 mechanisms include changes in abundances that affect predator-prey encounter probabilities

238 (Poisot et al., 2015), and prey behavioral changes causing prey to be less vulnerable given high

239 predator densities (Charnov, 1976; Skalski and Gilliam, 2001). Thus, all these pieces of evidence

240 suggest that species co-occurrence is necessary but not the only factor that influences the

241 occurrence of ecological interactions (Lopez et al., 2017; Poisot et al., 2012; Thompson and

242 Townsend, 1999).

243 Canard et al. (2011) and Poisot et al. (2012) developed a similar approach based on $\beta$ -

244 diversity measure of dissimilarity, traditionally used to assess community similarity. When

245 comparing these methods with the approach presented here, results showed that both approaches 
246 do not seem to share information (as reflected in AMI values). This could suggest that, because

247 their different origin, both approaches are not measuring exactly the same dimension of

248 community similarity. However, GED values were significantly more related to $\mathrm{B}_{\mathrm{WN}}$ than to $\mathrm{B}_{\mathrm{ST}}$

249 despite the cost scenario. This is not surprising because, as it is calculated, $\mathrm{B}_{\mathrm{WN}}$ contains to $\mathrm{B}_{\mathrm{ST}}$,

250 so this apparent pattern is just a consequence of $\mathrm{B}_{\mathrm{ST}}$ being a subset of $\mathrm{B}_{\mathrm{WN}}$. In this context, we

251 think that a valuable advantage of using GED is the flexibility of assigning different degrees of

252 importance to species or interactions in the network through differential costs for each type of

253 edition (Bunke and Allermann, 1983; Emmert-Streib et al., 2016). This characteristic of GED

254 allows researchers to focus the analysis into the specific components of their interest.

255 On the other hand, GED is an measure the similarity between pairwise networks that

256 incorporates both species composition and the structure of interactions, this implies that GED

257 includes compositional metrics. In this vein, two communities with high values of Jaccard would

258 be also similar in functionality (high GED or similar paths of energy flows) if and only if the

259 occurrence of the species is strongly related to the occurrence of the interactions.

260 Recently, other authors have proposed a different approach to integrate compositional

261 and "interactional" community similarities (Schmidt et al., 2017). In this approach, species co-

262 occurrences are used to infer biotic interactions, through similarity-based network inference.

263 This kind of inference includes constructing a network from pairwise co-occurrences and/or

264 mutual exclusion, and some metric is used to quantify and determine the significance of the

265 similarity of the pairwise distributions (Faust and Raes, 2012; Schmidt et al., 2017). Despite that

266 these methods have been successfully applied in microbial ecology, and their use has

267 complemented the information provided by standard analytical approaches (Barberán et al.,

268 2012, Cazelles et al., 2016, Faust and Raes, 2012; Freilich et al. 2018; Morales-Castilla et al., 
269 2015; Schmidt et al., 2017), the utility of this method to analyze interactions at macro-scales is

270 questionable, mainly due to the independence between co-occurrence measures and interactions

271 (Akin and Winemiller, 2006; Lopez et al., 2017; Poisot et al., 2012; Saavedra et al., 2016;

272 Thompson and Townsend, 1999). Some key restrictions of this theoretical framework have been

273 associated to its inability to exactly reproduce the interaction networks (Freilich et al. 2018;

274 Morales-Castilla et al., 2015), and that co-occurrences alone are not sufficient to provide insight

275 about the biotic interactions in these communities. As such, several have noted the importance of

276 directly collecting information of trophic interactions when analyzing natural communities

277 (Cazelles et al., 2016; Morales-Castilla et al., 2015,). This being said, collecting information

278 about trophic interactions is expensive and time-consuming, so in many cases the

279 implementation of this kind of sampling is not possible. Overall, given that metrics used to

280 describe communities do not necessarily share significant levels of information, researchers

281 should clearly identify not only their research question, but also the metrics (richness,

282 composition, evenness, abundance, interactions) that best describe the dimension of the

283 community to be analyzed.

284 The method used in this study reveals the effect that trophic interactions have on

285 communities similarity and highlight the constrains at analyzing community similarity in one or

286 few ecological dimensions. Similarly, others have developed a new approach to separate the

287 effects of interactions in ecosystem functioning from those of species composition (Jaillard et al.,

288 2018). These authors have found that the effects of interactions and composition are

289 independent, but both contribute significantly to ecosystem functioning. Thus, the direct

290 interpretation of species interactions from co-occurrence data remains controversial (Cazelles et

291 al., 2016). In our case, and unlike microbial ecology studies, it was not necessary to infer trophic 
292 interactions because they were directly measured. Furthermore, here the AMI values confirmed

293 that species co-occurrence and interactions did not equally portray the similarity of the

294 communities regardless of the different cost scenarios assigned to the species and the structure of

295 the interaction network.

296 The data analysis used in this study could be useful when interactions between species

297 should be inferred from co-occurrence data. In these cases, potential bias from assumed

298 relationship between composition and interactions could be avoided by attributing different costs

299 when calculating GED. For example, the impact of interactions similarities on the whole

300 similarity could be approximated by assigning different costs to link editions. If the whole

301 similarity is notoriously affected by link edition costs then co-occurrences and interactions are

302 likely independent. Because the selection of a particular cost scenario could be considered as

303 subjective, and so affecting the global result of the analysis, we think that following some simple

304 criteria this subjectiveness can be reduced. In our case and following the point of view of an

305 ecologist, the deletion of a node in a network can reflect the temporal extinction of a species, and

306 an addition reflects the opposite. The same can be applied to links that represent interactions. We

307 considered that deleting or adding links to a network has a lower cost due to the high variability

308 of the analyzed trophic interactions over time (Lopez et al., 2017), and because the presence of

309 an interaction depends hierarchically on species composition (a given interaction occurs if and

310 only if both interacting species co-occur). The flipping of an interaction was attributed a higher

311 cost, because this edition changes the hierarchy of the consumer-resource interactions and the

312 direction of energy transfer within the network, which is an ecologically costly and rare

313 phenomenon. 
In the ecological literature some level of association between state variables like richness,

315 abundances, species composition, or biomass is usually assumed; however, many of these

316 variables does not show robust association patterns (Edwards and Richardson, 2004; Poisot et al.,

317 2012; Pool et al., 2016). The poor association between the similarity indices shown in this study

318 reinforces the idea that each state variable represents a different dimension of natural systems.

319 Future studies could take advantages of methods like those used in this study to ensure the robust 320 assessment of the similitude of natural systems.

322 5. Conclusions

In summary, our results show: a) a weak relationship between measures of similarity

324 using only the species composition and that those include composition and interactions, evidence

325 the need of adding structural relationships to the similarity measure and b) GED can be

326 advantageous in the analysis of networks and ecological communities due to its flexibility in

327 assigning different cost schemes depending of the researcher interest. A more practical

328 consequence of our results is a cautionary note on community-level interpretation of similarity.

329 In the analysis of ecological communities, it is commonly assumed that different sites,

330 assemblages, or communities can be considered as replicates. However, the question that arises

331 is how similar must communities be in terms of any state variable to represent an adequate

332 replicate of the system?

333 Finally, Our results show that graph edit distance (GED) might be a valuable metric for

334 the analysis of ecological communities. The use of an integrated measure that allows us to

335 incorporate information of the composition and interaction structure of communities is useful for

336 effectively establishing whether two communities, rather than operational units, could be 
337 considered as equivalent ecological systems. Our results further give warning about the need to

338 account for the particularities of the multiple state variables that represent dimensions of

339 ecological systems.

340

341 Acknowledgements

342

343

344

345

346

347

348

349

350

351

352

353

354

355

356

357

358

359

360

361

362

363

364

365

366

367

We thank to Interaction Web Database of National Center for Ecological Analysis and Synthesis (https://www.nceas.ucsb.edu/interactionweb/), at the University of California, Santa Barbara, U.S.A.

References

Abrams PA. 1982. Functional responses of optimal foragers. American Naturalist 120(3): 382390. https://doi.org/10.1086/283996.

Akin S, Winemiller KO. 2006. Seasonal variation in food web composition and structure in a temperate tidal estuary. Estuaries and Coasts 29(4): 552-567. https://doi.org/10.1007/BF02784282.

Anderson MJ, Ellingsen KE, McArdle BH. 2006. Multivariate dispersion as a measure of beta diversity. Ecology letters. 9(6): 683-693. https://doi.org/10.1111/j.14610248.2006.00926.x.

Arditi R, Ginzburg LR. 2012. How species interact: altering the standard view on trophic ecology. Oxford University Press. https://doi.org/10.1086/669300.

Barberán A, Bates ST, Casamayor E, Fierer N. 2012. Using network analysis to explore cooccurrence patterns in soil microbial communities. Multidisciplinary Journal of Microbial Ecology 6(2): 343-351. doi:10.1038/ismej.2011.119.

Barwell LJ, Isaac NJB, Kunin WE. 2015. Measuring $\beta$-diversity with species abundance data. Journal of Animal Ecology 84(4): 1112-1122. https://doi.org/10.1111/1365-2656.12362.

Baselga A. 2012. The relationship between species replacement, dissimilarity derived from nestedness, and nestedness. Global ecology and biogeography 21(12): 1223-1232. https://doi.org/10.1111/j.1466-8238.2011.00756.x.

Baselga A. 2010. Partitioning the turnover and nestedness components of beta diversity. Global ecology and biogeography 19(1): 134-143. https://doi.org/10.1111/j.14668238.2009.00490.x.

Peer] reviewing PDF | (2018:08:30219:3:0:NEW 22 Apr 2019) 
368

369

370

371

372

373

374

375

376

377

378

379

380

381

382

383

384

385

386

387

388

389

390

391

392

393

394

395

396

397

398

399

400

401

Bezemer TM, Fountain MT, Barea JM, Christensen S, Dekker SC, Duyts H, Van Hal R, Harvey JA, Hedlund K, Maraun M. 2010. Divergent composition but similar function of soil food webs of individual plants: plant species and community effects. Ecology 91(10): 30273036. https://doi.org/10.1890/09-2198.1.

Bunke H. 1983. What is the distance between graphs. Bulletin of the European Association for Theoretical Computer Science 20: 35-39.

Bunke H, Allermann G. 1983. Inexact graph matching for structural pattern recognition. Pattern Recognition Letters 1(4): 245-253. https://doi.org/10.1016/0167-8655(83)90033-8.

Burkle LA, Marlin JC, Knight TM. 2013. Plant-pollinator interactions over 120 years: loss of species, co-occurrence, and function. Science 339 (6127): 1611-1615. https://doi.org/10.1126/science.1232728.

Canard E. 2011. Espace et neutralite dans les reseaux d' interactions ecologiques. PhD thesis. Universite Montpellier 2.

Cazelles K, Araújo MB, Mouquet N, Gravel D. 2016. A theory for species co-occurrence in interaction networks. Theoretical Ecology 9(1): 39-48. https://doi.org/10.1007/s12080-0150281-9

Charnov EL. 1976. Optimal foraging, the marginal value theorem. Theoretical Population Biology 9: 129-136. https://doi.org/10.1016/0040-5809(76)90040-X.

Dehmer M. 2010. Structural analysis of complex networks. Springer Science \& Business Media. https://doi.org/10.1007/978-0-8176-4789-6.

Devictor V, Mouillot D, Meynard C, Jiguet F, Thuiller W, Mouquet N. 2010. Spatial mismatch and congruence between taxonomic, phylogenetic and functional diversity: the need for integrative conservation strategies in a changing world. Ecology letters 13 (8): 1030-1040. https://doi.org/10.1111/j.1461-0248.2010.01493.x.

Edwards M, Richardson AJ. 2004. Impact of climate change on marine pelagic phenology and trophic mismatch. Nature 430(7002): 881. https://doi.org/10.1038/nature02808.

Emmert-Streib F, Dehmer M, Shi Y. 2016. Fifty years of graph matching, network alignment and network comparison. Information Sciences (Ny). 346: 180-197. https://doi.org/10.1016/j.ins.2016.01.074.

Faust K, Raes J. 2012. Microbial interactions: from networks to models. Nature Reviews Microbiology 10(8): 538. doi:10.1038/nrmicro2832.

Freilich MA, Wieters E, Broitman BR, Marquet PA, Navarrete SA. 2018. Species co-occurrence networks: Can they reveal trophic and non-trophic interactions in ecological communities?. Ecology 99(3): 690-699. https://doi.org/10.1002/ecy.2142. 
402 Harrington R, Woiwod I, Sparks T. 1999. Climate change and trophic interactions. Trends in 403 Ecology \& Evolution 14(4): 146-150. https://doi.org/10.1016/S0169-5347(99)01604-3.

404 Ibragimov R, Malek M, Guo J, Baumbach J. 2013. GEDEVO: an evolutionary graph edit 405 distance algorithm for biological network alignment. In: OASIcs-OpenAccess Series in 406 Informatics. Schloss Dagstuhl-Leibniz-Zentrum fuer Informatik.

407 https://doi.org/10.4230/OASIcs.GCB.2013.68.

408

409

410

411

412

413

414

415

416

417

418

419

420

421

422

423

424

425

426

427

428

429

430

431

432

433

434

Jaarsma NG, De Boer SM, Townsend CR, Thompson RM, Edwards ED. 1998. Characterising food-webs in two New Zealand streams. New Zealand Journal of Marine and Freshwater Research 32(2): 271-286. https://doi.org/10.1080/00288330.1998.9516825.

Jaillard B, Richon C, Deleporte P, Loreau M, Violle C. 2018. An a posteriori species clustering for quantifying the effects of species interactions on ecosystem functioning. Methods in Ecology and Evolution 9(3): 704-715. https://doi.org/10.1111/2041-210X.12920.

Koleff P, Gaston KJ, Lennon JJ. 2003. Measuring beta diversity for presence-absence data. Journal of Animal Ecology 72(3): 367-382. https://doi.org/10.1046/j.13652656.2003.00710.x.

Lopez DN, Camus PA, Valdivia N, Estay SA. 2017. High temporal variability in the occurrence of consumer-resource interactions in ecological networks. 126(12): 1699-1707.

DOI10.1111/oik.04285.

Malek M. 2015. CytoGEDEVO: A Cytoscape app for fast and interactive network alignment. Master Thesis, Saarland University. Germany. 82 pp.

Meyer PE.2014. Infotheo: Information-Theoretic Measures. URL http://CRAN. R-project. org/package $=$ infotheo. $\mathrm{R}$ package version, $1(0)$.

Morales-Castilla I, Matias MG, Gravel D, Araújo MB. 2015. Inferring biotic interactions from proxies. Trends in Ecology \& Evolution, 30(6): 347-356. https://doi.org/10.1016/j.tree.2015.03.014 .

Morlon H, Kefi S, Martinez ND. 2014. Effects of trophic similarity on community composition. Ecology letters 17(12): 1495-1506. https://doi.org/10.1111/ele.12356.

Neyshabur B, Khadem A, Hashemifar S, Arab SS. 2013. NETAL: a new graph-based method for global alignment of protein-protein interaction networks. Bioinformatics 29(13): 16541662. https://doi.org/10.1093/bioinformatics/btt202.

Oksanen J, Blanchet FG, Michael F, Kindt R, Legendre P, McGlinn DP, Minchin PR, O'hara RB, Simpson G, Solymos P, Stevens MH, Szoecs E, Wagner H. 2013. Package 'vegan'. Community ecology package, version, 2(9). 
435 Olden JD, Poff NL, Douglas MR, Douglas ME, Fausch KD. 2004. Ecological and evolutionary 436 consequences of biotic homogenization. Trends in Ecology and Evolution 19(1): 18-24. 437 https://doi.org/10.1016/j.tree.2003.09.010

438 R Development Core Team 2016 R: A language and environment for statistical computing. 439 Vienna, Austria: R Foundation for Statistical Computing.

440 Petchey OL, Gaston KJ. 2006. Functional diversity: back to basics and looking forward. Ecology letters 9(6): 741-758. https://doi.org/10.1111/j.1461-0248.2006.00924.x.

Poisot T, Canard E, Mouillot D, Mouquet N, Gravel D, Jordan F. 2012. The dissimilarity of species interaction networks. Ecology letters 15(12): 1353-61. https://doi.org/10.1111/ele.12002

Poisot T, Stouffer DB, Gravel D. 2015. Beyond species: why ecological interaction networks vary through space and time. Oikos 124(3): 243-251. https://doi.org/10.1111/oik.01719

447

448

449

450

451

452

453

454

455

456

457

458

459

460

461

462

463

464

465

466

467

Pool TK, Cucherousset J, Boulêtreau S, Villéger S, Strecker AL, Grenouillet G. 2016. Increased taxonomic and functional similarity does not increase the trophic similarity of communities. Global ecology and biogeography 25(1): 46-54. https://doi.org/10.1111/geb.12384

Riesen K. 2016. Structural Pattern Recognition with Graph Edit Distance: Approximation Algorithms and Applications. Springer. https://doi.org/10.1007/978-3-319-27252-8

Saavedra S, Rohr RP, Fortuna MA, Selva N, Bascompte J. 2016. Seasonal species interactions minimize the impact of species turnover on the likelihood of community persistence. Ecology 97(4): 865-873. https://doi.org/10.1890/15-1013.1.

Schmidt TSB, Rodrigues JFM, Von Mering C. 2017. A family of interaction-adjusted indices of community similarity. Multidisciplinary Journal of Microbial Ecology 11(3): 791. https://doi.org/10.1890/15-1013.1.

Shannon P, Markiel A, Ozier O, Baliga NS, Wang JT, Ramage D, Amin N, Schwikowski B, Ideker T. 2003. Cytoscape: a software environment for integrated models of biomolecular interaction networks. Genome research 13(11): 2498-2504. https://doi.org/10.1101/gr.1239303

Skalski GT, Gilliam JF. 2001. Functional responses with predator interference: viable alternatives to the holling type II model. Ecology 82(11): 3083-3092. https://doi.org/10.1890/0012-9658(2001)082[3083:FRWPIV]2.0.CO;2

Solé-Ribalta A, Serratosa F, Sanfeliu A. 2012. On the graph edit distance cost: properties and applications. International Journal of Pattern Recognition and Artificial Intelligence 26(05): 1260004. https://doi.org/10.1142/S021800141260004X 
468

469

470

471

472

473

474

475

476

477

478

479

480

481

482

483

484

485

486

487

488

489

490

491

492

493

494

495

496

497

498

499

500

501

Strona G, Veech JA. 2015. A new measure of ecological network structure based on node overlap and segregation. Methods in Ecology and Evolution 6(8): 907-915. https://doi.org/10.1111/2041-210X.12395

Strona G, Matthews TJ, Kortsch S, Veech JA. 2018. NOS: a software suite to compute node overlap and segregation in ecological networks. Ecography 41(3), 558-566.

Thackeray SJ, Sparks TH, Frederiksen M, Burthe S, Bacon PJ, Bell JR, Botham MS, Brereton T, Bright PW, Carvalho L, Clutton-Brock TIM, Dawson A, Edwards M, Elliott JM, Harrington R, Johns D, Jones I, Jones JT, Leech DI, Roy DB, Scott WA, Smith M, Smithers RJ, Winfield I, Wanless S. 2010. Trophic level asynchrony in rates of phenological change for marine, freshwater and terrestrial environments. Global Change Biology 16(12): 33043313. https://doi.org/10.1111/j.1365-2486.2010.02165.x

Thompson RM, Townsend CR. 2005. Energy availability, spatial heterogeneity and ecosystem size predict food-web structure in streams. Oikos 108(1): 137-148. https://doi.org/10.1111/j.0030-1299.2005.11600.x.

Thompson RM, Townsend CR. 2003. Impacts on stream food webs of native and exotic forest: an intercontinental comparison. Ecology 84(1): 145-161. https://doi.org/10.1890/00129658(2003)084[0145:IOSFWO]2.0.CO;2.

Thompson RM, Townsend CR. 2000. Is resolution the solution? the effect of taxonomic resolution of three stream food webs on the calculated properties. Freshwater Biology 44(3): 413-422. https://doi.org/10.1046/j.1365-2427.2000.00579.x

Thompson RM, Townsend CR. 1999. The effect of seasonal variation on the community structure and food-web attributes of two streams: implications for food-web science. Oikos 87, 75-88. https://doi.org/10.2307/3546998

Trøjelsgaard K, Jordano P, Carstensen DW, Olesen JM. 2015. Geographical variation in mutualistic networks: similarity, turnover and partner fidelity. Proceedings of the Royal Society B: Biological Sciences, 282(1802), 20142925. https://doi.org/10.1098/rspb.2014.2925.

Vinh NX, Epps J, Bailey J. 2010. Information theoretic measures for clusterings comparison: Variants, properties, normalization and correction for chance. Journal of Machine Learning Research 11, 2837-2854. https://doi.org/10.1145/1553374.1553511.

Whittaker RH. 1972. Evolution and measurement of species diversity. Taxon 213-251. https://doi.org/10.2307/1218190.

Whittaker RH. 1960. Vegetation of the Siskiyou mountains, Oregon and California. Ecological monographs 30(3): 279-338. https://doi.org/10.2307/1943563. 
502 Wilson MV, Shmida A. 1984. Measuring beta diversity with presence-absence data. The Journal 503 of Ecology 1055-1064. https://doi.org/10.2307/2259551.

504 Zhang S, Liu H, Ning X, Zhang X, Xu Y, Liu P, Li X, Ren W, Van Mieghem P, Omic J, Poulin 505 R, Montoya JM, Sole RV, Lu X, Gray C, Brown LE, Ledger ME, Milner AM, Mondragón 506 RJ, Woodward G, Ma A, Lü L, Chen D, Ren XL, Zhang QM, Zhang YC, Zhou T, Lo CM, 507 Morand S, Galzin R, Estudio P, Arneberg P, Skorping A, Grenfell B, Read AF. 2016. Vital 508 nodes identification in complex networks. Physics Reports 650, 1-63.

509 https://doi.org/10.1155/2014/407639.

510

511 
Figure 1 (on next page)

Relationship between the similitude in species composition (1-Jaccard) and similarity of interactions (1-GED) in grassland systems

A. Flipping:0.75, Add or delete nodes:1, Add or delete links: 0, B. Flipping:0.75, Add or delete nodes:0.75, Add or delete links: 0.25, C. Flipping:0.75, Add or delete nodes:0.25, Add or delete links: 0.75 , D. Flipping:0.25, Add or delete nodes:0, Add or delete links:1. AMI values are included in each plot. Each point represents a pairwise comparison between food webs. 
Figure 2 (on next page)

AMI values of relationship between the similitude in species composition (1-Jaccard) and similarity of interactions (1-GED) in grassland systems. Color codes shows the AMI values. A. Flipping 0.25 , B. Flipping 0.75 


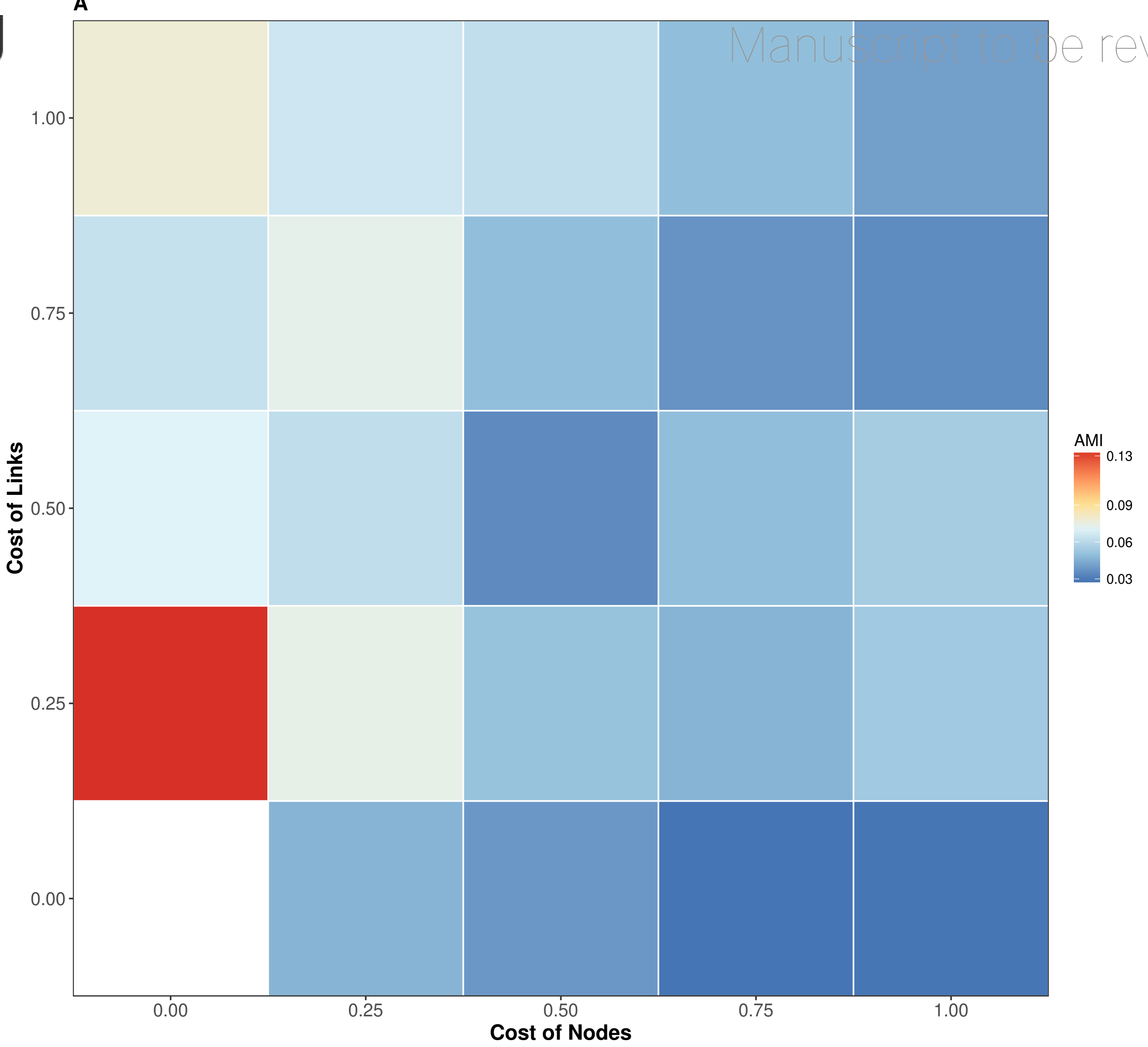

B

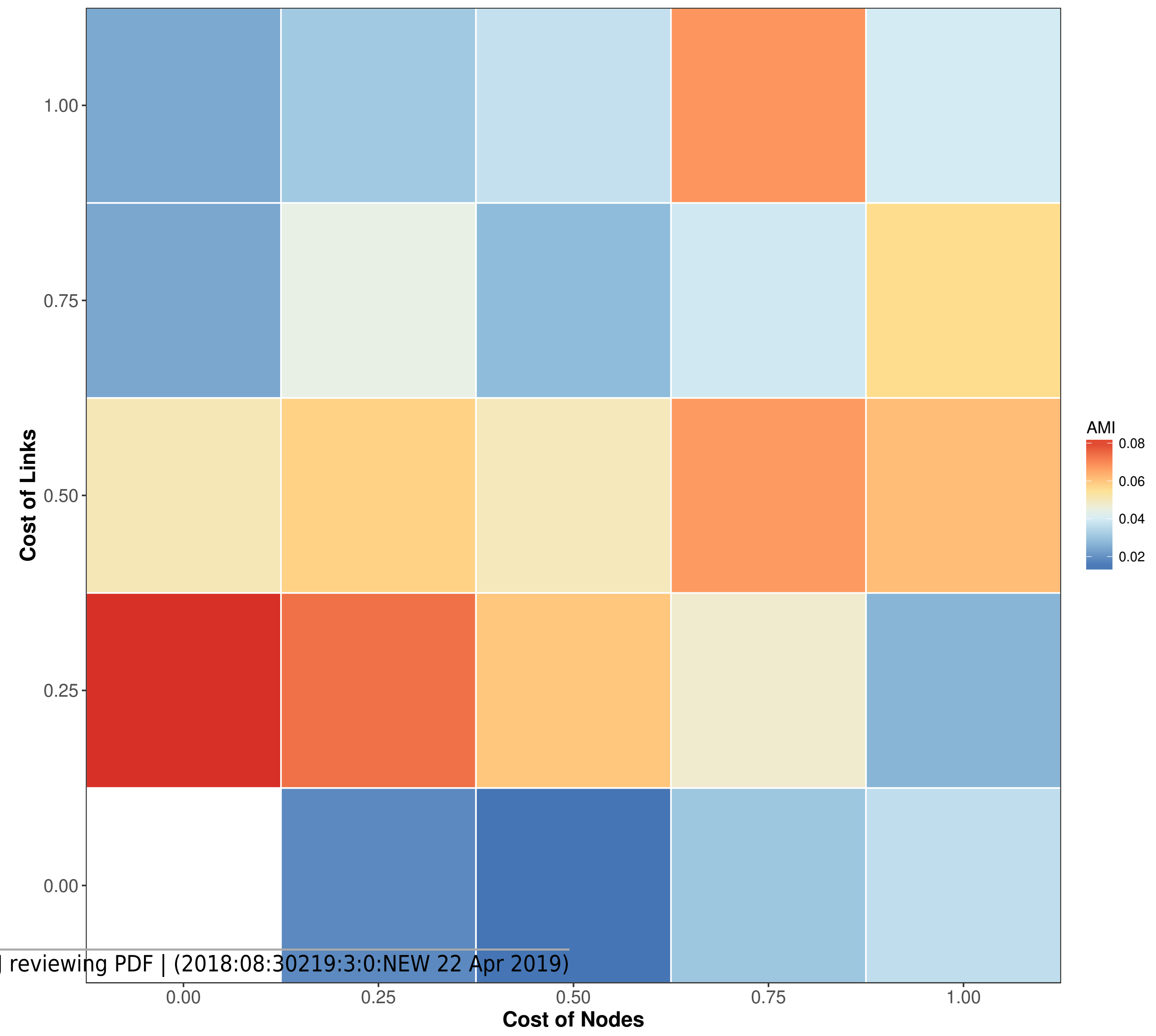


Figure 3 (on next page)

Relationship among the dissimilarity of interactions due to species turnover $\left(\mathrm{B}_{\mathrm{ST}}\right)$, dissimilarity of interactions $\left(B_{w N}\right)$ and Graph Edit Distance (1-GED) in grassland systems A. Flipping:0.75, Add or delete nodes:1, Add or delete links: 0,25, B. Flipping:0.75, Add or delete nodes:1, Add or delete links: 0.50, C. Flipping:0.75, Add or delete nodes:0.25, Add or delete links: 0.25, D. Flipping:0.75, Add or delete nodes:0.25, Add or delete links:1 
PeerJ

A

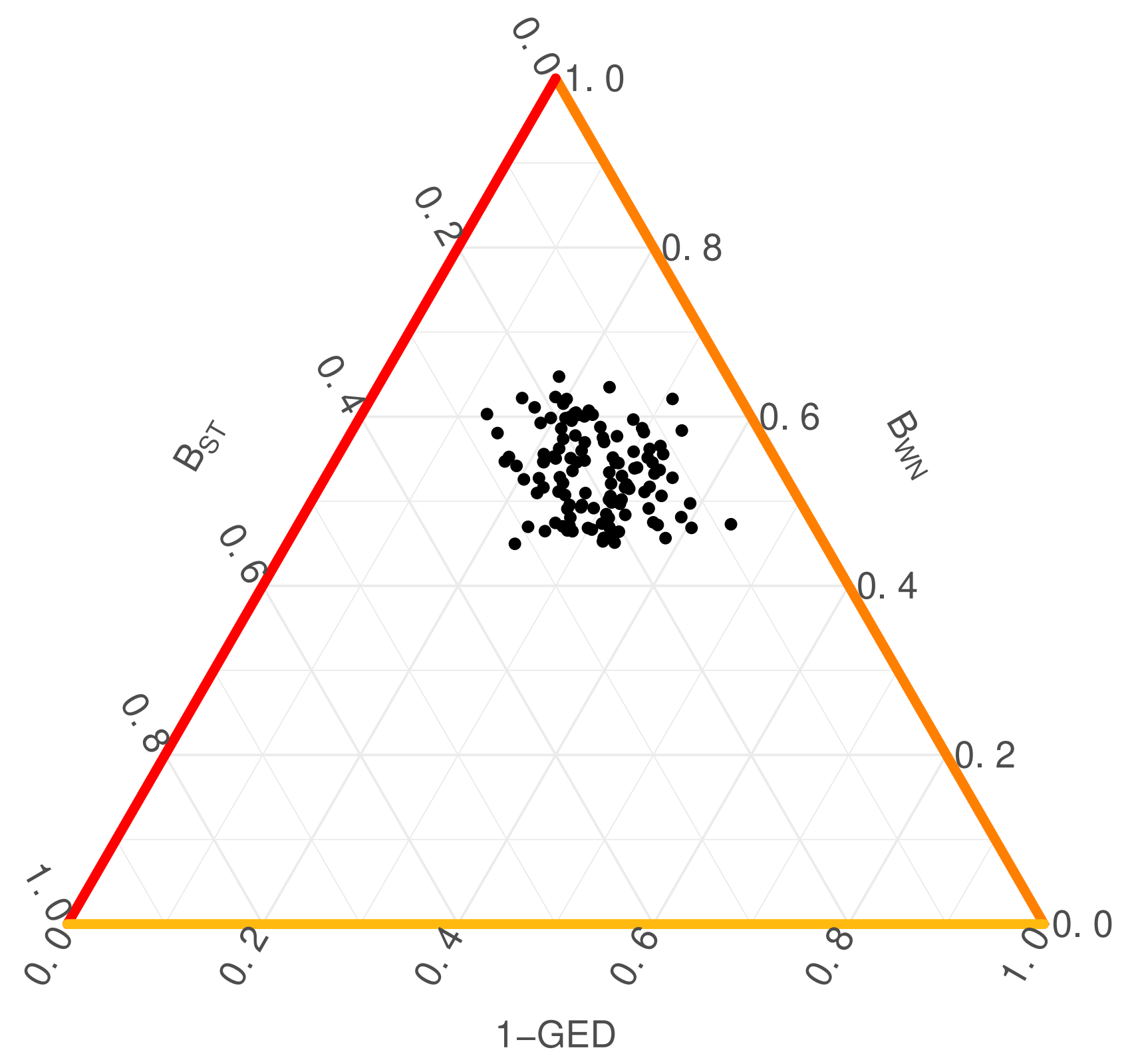

C

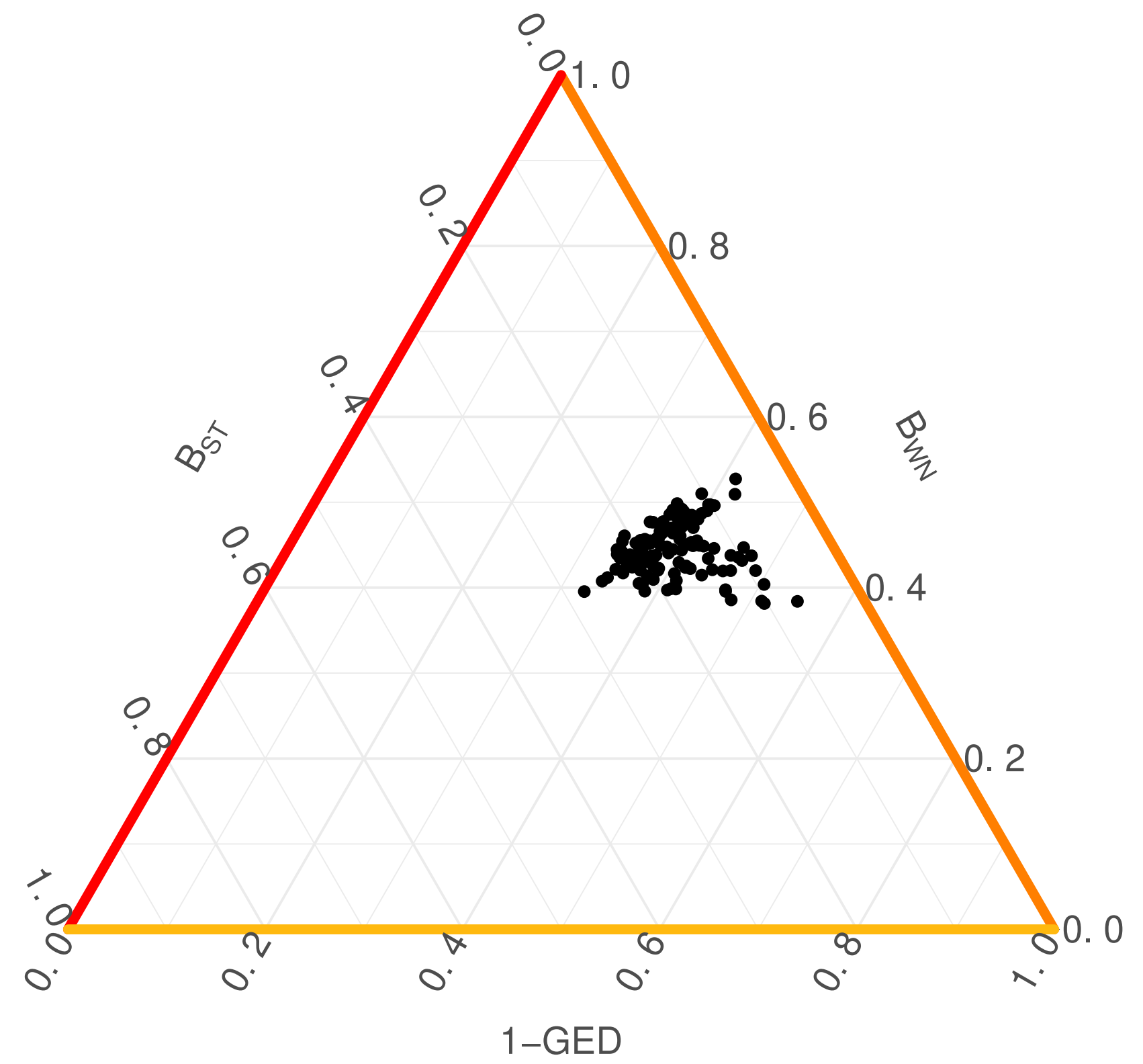

Manuscript to be reviewed

B

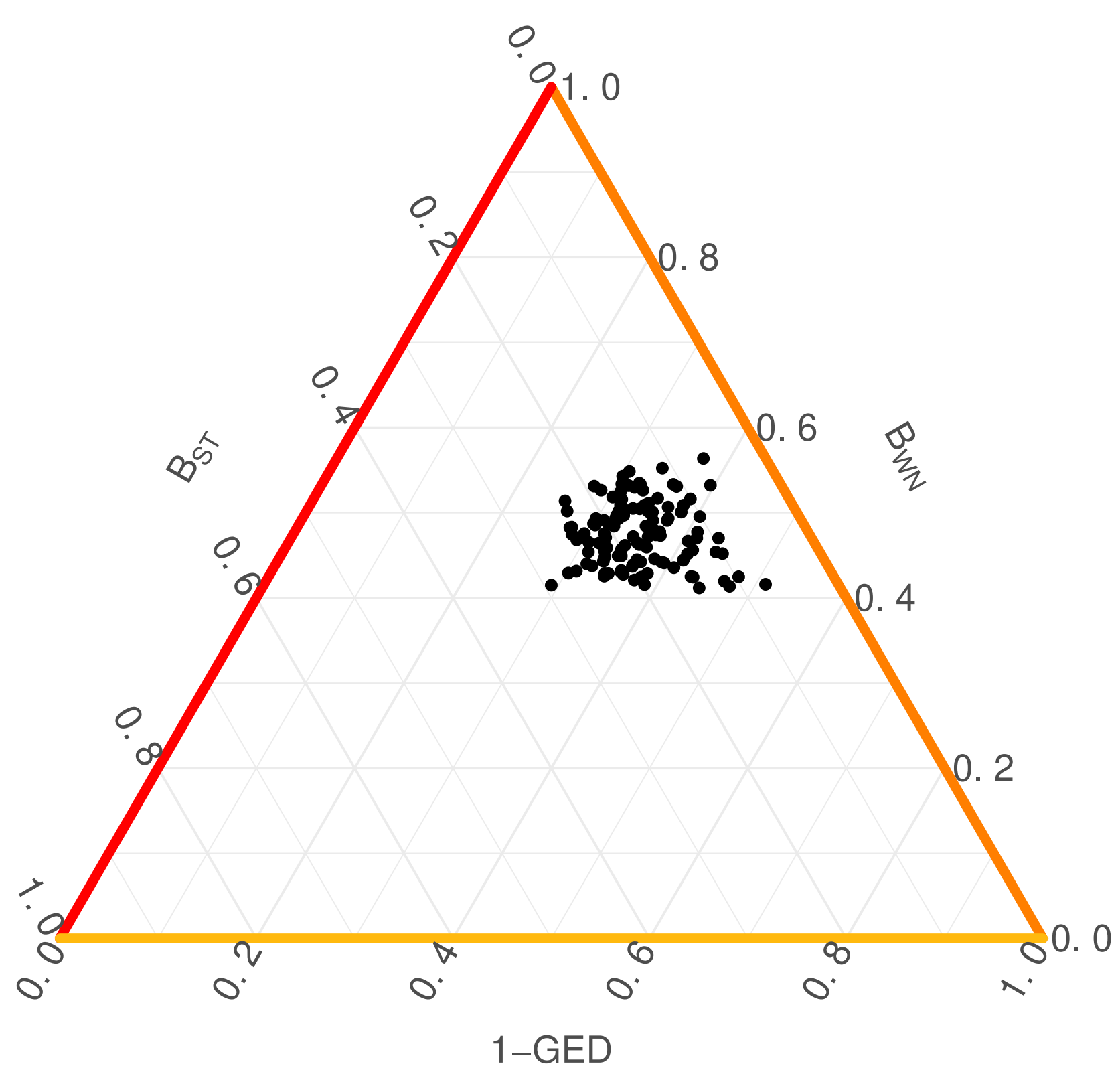

D

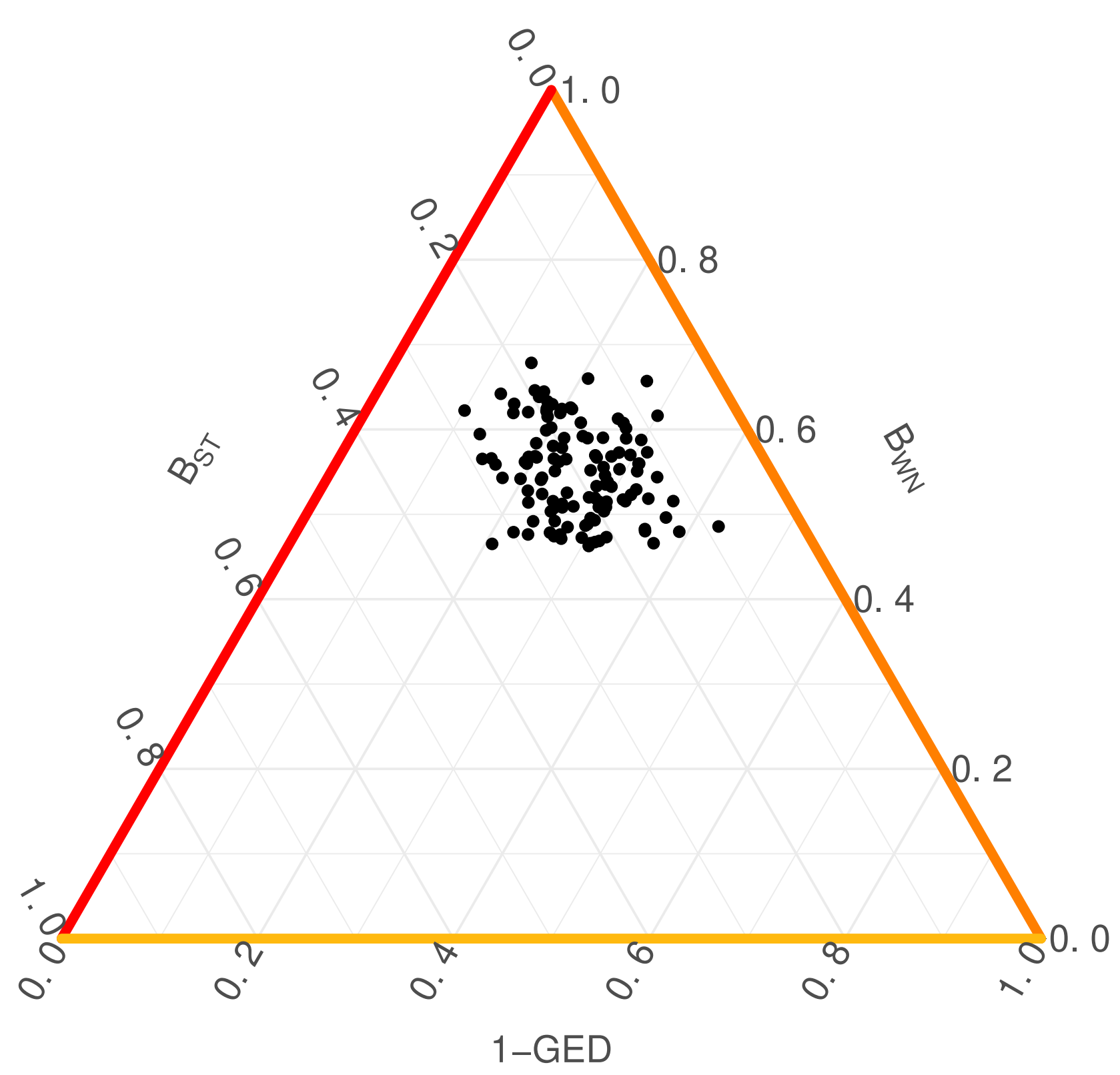




\section{Table $\mathbf{1}$ (on next page)}

Scenarios of editing costs, AMI values of relationship between the similitude in species composition (1-Jaccard) and similarity of interactions (1-GED) 


\begin{tabular}{|c|c|c|c|c|}
\hline Scenarios & $\begin{array}{l}\text { Flipping an } \\
\text { interaction }\end{array}$ & $\begin{array}{l}\text { Deleting or } \\
\text { adding a species }\end{array}$ & Deleting or adding an interaction & $\begin{array}{l}\text { AMI }_{(1-G E D, ~ 1-} \\
\text { Jaccard) }\end{array}$ \\
\hline 1 & 1 & 1 & 1 & 0.032 \\
\hline 2 & 0.25 & 0 & 0.25 & 0.134 \\
\hline 3 & 0.25 & 0 & 0.5 & 0.071 \\
\hline 4 & 0.25 & 0 & 0.75 & 0.064 \\
\hline 5 & 0.25 & 0 & 1 & 0.078 \\
\hline 6 & 0.25 & 0.25 & 0 & 0.047 \\
\hline 7 & 0.25 & 0.25 & 0.25 & 0.074 \\
\hline 8 & 0.25 & 0.25 & 0.5 & 0.062 \\
\hline 9 & 0.25 & 0.25 & 0.75 & 0.073 \\
\hline 10 & 0.25 & 0.25 & 1 & 0.065 \\
\hline 11 & 0.25 & 0.5 & 0 & 0.039 \\
\hline 12 & 0.25 & 0.5 & 0.25 & 0.051 \\
\hline 13 & 0.25 & 0.5 & 0.5 & 0.035 \\
\hline 14 & 0.25 & 0.5 & 0.75 & 0.05 \\
\hline 15 & 0.25 & 0.5 & 1 & 0.062 \\
\hline 16 & 0.25 & 0.75 & 0 & 0.029 \\
\hline 17 & 0.25 & 0.75 & 0.25 & 0.047 \\
\hline 18 & 0.25 & 0.75 & 0.5 & 0.05 \\
\hline 19 & 0.25 & 0.75 & 0.75 & 0.037 \\
\hline 20 & 0.25 & 0.75 & 1 & 0.05 \\
\hline 21 & 0.25 & 1 & 0 & 0.029 \\
\hline 22 & 0.25 & 1 & 0.25 & 0.054 \\
\hline 23 & 0.25 & 1 & 0.5 & 0.055 \\
\hline 24 & 0.25 & 1 & 0.75 & 0.035 \\
\hline 25 & 0.25 & 1 & 1 & 0.041 \\
\hline 26 & 0.75 & 0 & 0.25 & 0.084 \\
\hline 27 & 0.75 & 0 & 0.5 & 0.051 \\
\hline 28 & 0.75 & 0 & 0.75 & 0.024 \\
\hline 29 & 0.75 & 0 & 1 & 0.024 \\
\hline 30 & 0.75 & 0.25 & 0 & 0.018 \\
\hline
\end{tabular}




\begin{tabular}{|c|c|c|c|c|}
\hline 31 & 0.75 & 0.25 & 0.25 & 0.074 \\
\hline 32 & 0.75 & 0.25 & 0.5 & 0.058 \\
\hline 33 & 0.75 & 0.25 & 0.75 & 0.044 \\
\hline 34 & 0.75 & 0.25 & 1 & 0.031 \\
\hline 35 & 0.75 & 0.5 & 0 & 0.014 \\
\hline 36 & 0.75 & 0.5 & 0.25 & 0.06 \\
\hline 37 & 0.75 & 0.5 & 0.5 & 0.05 \\
\hline 38 & 0.75 & 0.5 & 0.75 & 0.027 \\
\hline 39 & 0.75 & 0.5 & 1 & 0.037 \\
\hline 40 & 0.75 & 0.75 & 0 & 0.03 \\
\hline 41 & 0.75 & 0.75 & 0.25 & 0.047 \\
\hline 42 & 0.75 & 0.75 & 0.5 & 0.068 \\
\hline 43 & 0.75 & 0.75 & 0.75 & 0.039 \\
\hline 44 & 0.75 & 0.75 & 1 & 0.068 \\
\hline 45 & 0.75 & 1 & 0 & 0.036 \\
\hline 46 & 0.75 & 1 & 0.25 & 0.026 \\
\hline 47 & 0.75 & 1 & 0.5 & 0.062 \\
\hline 48 & 0.75 & 1 & 0.75 & 0.056 \\
\hline 49 & 0.75 & 1 & 1 & 0.04 \\
\hline
\end{tabular}




\section{Table 2 (on next page)}

Scenarios of editing cost, AMI values of relationship between the dissimilarity of interactions $\left(B_{W N}\right)$, dissimilarity of interactions due to species turnover $\left(B_{S T}\right)$ and similarity of interactions (1-GED). 


\begin{tabular}{|c|c|c|c|c|c|}
\hline Scenarios & $\begin{array}{l}\text { Flipping an } \\
\text { interaction }\end{array}$ & $\begin{array}{l}\text { Deleting or } \\
\text { adding a species }\end{array}$ & $\begin{array}{l}\text { Deleting or } \\
\text { adding an interaction }\end{array}$ & $\begin{array}{l}\text { AMI } \\
\left(1-G E D . B_{S T}\right)\end{array}$ & $\begin{array}{l}\text { AMI } \\
\left(1-G E D . B_{W N}\right)\end{array}$ \\
\hline 1 & 1 & 1 & 1 & $\sim 0$ & 0.017 \\
\hline 2 & 0.25 & 0 & 0.25 & 0.014 & 0.156 \\
\hline 3 & 0.25 & 0 & 0.5 & 0.025 & 0.099 \\
\hline 4 & 0.25 & 0 & 0.75 & 0.027 & 0.083 \\
\hline 5 & 0.25 & 0 & 1 & 0.007 & 0.066 \\
\hline 6 & 0.25 & 0.25 & 0 & 0.001 & 0.034 \\
\hline 7 & 0.25 & 0.25 & 0.25 & 0.002 & 0.059 \\
\hline 8 & 0.25 & 0.25 & 0.5 & 0.029 & 0.102 \\
\hline 9 & 0.25 & 0.25 & 0.75 & 0.019 & 0.086 \\
\hline 10 & 0.25 & 0.25 & 1 & 0.023 & 0.071 \\
\hline 11 & 0.25 & 0.5 & 0 & $\sim 0$ & 0.025 \\
\hline 12 & 0.25 & 0.5 & 0.25 & $\sim 0$ & 0.025 \\
\hline 13 & 0.25 & 0.5 & 0.5 & 0.004 & 0.013 \\
\hline 14 & 0.25 & 0.5 & 0.75 & 0.002 & 0.056 \\
\hline 15 & 0.25 & 0.5 & 1 & 0.024 & 0.05 \\
\hline 16 & 0.25 & 0.75 & 0 & $\sim 0$ & 0.018 \\
\hline 17 & 0.25 & 0.75 & 0.25 & $\sim 0$ & 0.019 \\
\hline 18 & 0.25 & 0.75 & 0.5 & $\sim 0$ & 0.020 \\
\hline 19 & 0.25 & 0.75 & 0.75 & $\sim 0$ & 0.021 \\
\hline 20 & 0.25 & 0.75 & 1 & 0.015 & 0.049 \\
\hline 21 & 0.25 & 1 & 0 & 0.002 & 0.021 \\
\hline 22 & 0.25 & 1 & 0.25 & $\sim 0$ & 0.014 \\
\hline 23 & 0.25 & 1 & 0.5 & $\sim 0$ & 0.017 \\
\hline 24 & 0.25 & 1 & 0.75 & $\sim 0$ & 0.009 \\
\hline 25 & 0.25 & 1 & 1 & $\sim 0$ & 0.03 \\
\hline 26 & 0.75 & 0 & 0.25 & $\sim 0$ & 0.088 \\
\hline 27 & 0.75 & 0 & 0.5 & 0.006 & 0.045 \\
\hline 28 & 0.75 & 0 & 0.75 & 0.007 & 0.032 \\
\hline 29 & 0.75 & 0 & 1 & 0.01 & 0.009 \\
\hline 30 & 0.75 & 0.25 & 0 & 0.005 & 0.015 \\
\hline 31 & 0.75 & 0.25 & 0.25 & 0.015 & 0.068 \\
\hline 32 & 0.75 & 0.25 & 0.5 & 0.009 & 0.04 \\
\hline 33 & 0.75 & 0.25 & 0.75 & $\sim 0$ & 0.025 \\
\hline 34 & 0.75 & 0.25 & 1 & 0.007 & 0.037 \\
\hline 35 & 0.75 & 0.5 & 0 & $\sim 0$ & 0.019 \\
\hline 36 & 0.75 & 0.5 & 0.25 & $\sim 0$ & 0.055 \\
\hline
\end{tabular}




\begin{tabular}{llllll}
\hline 37 & 0.75 & 0.5 & 0.5 & 0.006 & 0.035 \\
\hline 38 & 0.75 & 0.5 & 0.75 & $\sim 0$ & 0.015 \\
\hline 39 & 0.75 & 0.5 & 1 & $\sim 0$ & 0.023 \\
\hline 40 & 0.75 & 0.75 & 0 & 0.011 & 0.029 \\
\hline 41 & 0.75 & 0.75 & 0.25 & 0.015 & 0.031 \\
\hline 42 & 0.75 & 0.75 & 0.5 & $\sim 0$ & 0.038 \\
\hline 44 & 0.75 & 0.75 & 0.75 & $\sim 0$ & 0.022 \\
\hline 45 & 0.75 & 0.75 & 1 & $\sim 0$ & 0.037 \\
\hline 46 & 0.75 & 1 & 0 & $\sim 0$ & 0.024 \\
\hline 47 & 0.75 & 1 & 0.25 & $\sim 0$ & 0.011 \\
\hline 48 & 0.75 & 1 & 0.50 & $\sim 0$ & 0.032 \\
\hline 49 & 0.75 & 1 & 0.75 & 0.006 & 0.036 \\
\hline
\end{tabular}

1 